life: (1) Surgical shock is less because the nervous system of a young child is not well developed. Hæmorrhage is slight, and all mental impressions are eliminated; (2) if the muscles are brought into action early they develop instead of undergoing atrophy, and hence a good velum is secured with plenty of tissue, whereas if operation be undertaken later the parts are so shrunken from disuse that they never properly recover; (3) the alveolar margin of the upper jaw is brought into its proper relation with that of the lower jaw, and the nasal cavities are restored to their proper form; (4) when the child learns to talk no nasal accent is developed. If the operation is not done until faulty habits of speech have been acquired it is with difficulty that they can be overcome. In cases where the cleft palate is associated with hare-lip, Dr. Brophy prefers to attack the palate first, as the surgeon then has more room for the necessary manipulation.

1 Brooklyn Med. Jour., Sept., 1903.

\section{POST-TYPHOID SEPSIS.}

Under this heading, Dr. Delafield ${ }^{1}$ discusses those cases where, after an attack of typhoid, a patient has fever which cannot be attributed to a relapse. $\mathrm{He}$ points out the importance of making a distinction between what may be considered as a complication of enteric fever and a relapse which is really a fresh attack of typhoid. Thus there may be inflammation of the gall-bladder, of the urinary bladder, of connective tissue-all due to infection with typhoid baccilli, but they are not typhoid fever. In the same way, after typhoid fever there are fevers which may be due to typhoid baccilli or to other infections, but they are not typhoid fever. Among these may be distinguished (1) the ordinary moderate daily rises of temperature, only lasting for a few hours, occurring within three weeks after the end of an attack of typhoid fever. These rises of temperature would probably be overlooked entirely unless the thermometer were systematically used, for they are accompanied by no symptoms and do no harm. The only necessary thing is to know that they are unimportant. (2) The post-typhoid fevers which are of real importance, and last for one or more weeks, but yet do not make the patient very ill and are not fatal. This form of fever occurs quite frequently. The rise of temperature may be continuous with the typhoid temperature, or there may be an interval of several days of normal temperature before the post-typhoid fever begins. The temperature is of remittent type, the rises sometimes being as high as $105^{\circ} \mathrm{F}$. With this fever there is no delirium or sleeplessness, the tongue is moist and clean, The patients eruption, no tympanites, no diarrbœa. they may are hungry, and if kept on low diet they may have rather alarming attacks of heart failure. As long as they are kept in bed and on a of the fever. out of bed and freed proper treatment is to get them recover bed and feed them up, when they rapidly recover strength and put on flesh. (3) More important than these mild fevers are the severe and long.continued post-typhoid fevers which may terminate fatally. They occur as a rule after severe cases of typhoid fever and the septic fever is continuous with the typhoid fever. The temperature is remittent, rising to $102^{\circ}$ or $103^{\circ}$ in the afternoon, with perhaps occasional sudden jumps to $105^{\circ}$ or $106^{\circ}$. The patient looks septic rather than in the typhoid state. The mind is often dull and apathetic, but there is no active delirium. The tongue is dry, there may be nausea and vomiting, and yet there is some desire for food. There is a rapid loss of flesh and strength so that the condition may become alarming. Thrombosis of one or both femoral veins may occur. If the patients are insufficiently fed they are very apt to die. In the autopsies of the fatal cases which he has made, Dr. Delafield has found the intestinal lesions healed, while no cause of death was apparent beyond sepsis and starvation.

In these cases, as in the milder forms, the correct treatment is to get the patients out of bed and give them plenty of food. Then they gain flesh and strength even while the fever continues. Such treatment will call for a good deal of courage on the part of the medical man in attendance on the patient, but Dr. Delafield is quite convinced as to the correctness and success of the method he suggests.

$$
1 \text { Medical Record. }
$$

\section{THE SURGICAL TREATMENT OF COLITIS.}

At the present time it is not usual to invoke the aid of surgery for the relief of colitis until medical treatment has had a prolonged trial and has failed. According to Dr. Gibson ${ }^{1}$ this is a mistake, and he urges that if surgery is to succeed where medicine has failed it should be made use of at an earlier stage of the disease, before the acute condition has led to such permanent alterations in the bowel as may preclude a favourable issue. This end, he admits, can be attained only if surgical art can offer methods giving a reasonable certainty of improvement, yet devoid of the risks and possible bad after-results inherent to a serious surgical operation. In his experience it is usual in bad cases of colitis of any origin to find the whole of the large intestine involved in the inflammatory process. Topical treatment from below is bound, therefore, to be inefficient, and in order to treat the whole bowel, in those cases which do not yield to gentler measures, the aid of the surgeon may be advantageously sought. Until recently the surgical treatment of colitis consisted of making an artificial anus in order to give rest to the bowel and for the treatment of the ulcerated areas by appropriate local applications. The objections to this operation, as Dr. Gibson points out, are manifold. In the first place a simple opening into the gut gives a maximum amount of discomfort, while perfect physiological rest for the bowel below can only be obtained by a complete artificial anus necessitating for its future repair one of the severest operations practised in abdominal surgery. With a view to adopting a middle course, Dr. Gibson devised a plan of creating an artificial valvular fistula into the cæcum. This he does after the manner of Kader's gastrostomy. By this method a fistula is formed which does not leak and which will close spontaneously as soon as the daily insertion of a tube is omitted. Topical applications can be commenced on the third day after the operation. The tube can be left out at the end of a week, after which it is inserted two or three times a day in order to 
irrigate the large bowel and to apply local medication.

Summing up the advantages of this operation, Dr. Gibson states that the results have been such as to justify the hope of marked amelioration or cure of the symptoms. It is devoid of every disagreeable feature of an artificial anus. It calls for only a short confinement to bed. If a prolonged course of treatment is required, it may be carried out if necessary by the patient himself. A change of climate, so often beneficial, is made possible. Its performance is perfectly simple and safe. The intermuscular incision greatly reduces the risk of a subsequent hernia. The prompt closure of the fistula is certain and spontaneous.

Whether Dr. Gibson's operation will stand the test of time and experience and become an approved prac. tice in cases of colitis remains of course to be seen, but it is certainly an ingenious and apparently rational method of treatment.

\section{Medical Record.}

\section{CEREBRO-SPINAL FLUID IN GENERAL PARALYSIS.}

Aвоuт a year ago Joffroy and Mercier ${ }^{1}$ drew attention to the fact that in general paralysis there is an augmentation of the number of white blood corpuscles in the cerebro-spinal fluid. They found that the phenomenon was almost always present in cases of general paralysis, and they came to the conclusion that the sign was a valuable aid to diagnosis in this disease. Increase of the number of leucocytes in the cerebro-spinal fluid, that is, above
2 per c.m., was also found in four cases of tabes dorsalis and in one case of syphilitic disease of the spinal cord. From 120 punctures made upon 91 patients the results were so constant that Joffroy and Mercier considered that general paralysis could be excluded if there was absence of leucocytosis in the cerebro-spinal fluid, and conversely that if there was leucocytosis general paralysis might be diagnosed provided that tabes dorsalis could be excluded. Dr. Jost D. Kramer ${ }^{2}$ has made further observations in the same direction. He examined 29 patients in all. Eleven of these had general paralysis, and the leucocyte count of the cerebro-spinal fluid showed an excess of leucocytes in every instance, the number per c.m. varying from six to as many as 145 in one case. The remaining 18 patients were suffering from various kinds of mental disease, including dementia præcox, Huntingdon's chorea, epileptic insanity, melancholia, chronic alcoholism, and pseudo-diabetic tabes. In none of them did the leucocytes exceed 2 per c.m. Dr. Kramer prefers to perform the lumbar puncture with the patient sitting upon a chair, or else with the patient lying on his side with the knees strongly flexed. The needle is inserted into the interspace between the fourth and fifth lumbar vertebræ a little to the right of the middle line. Dr. Kramer says that the patient shows very little discomfort at the time of the operation, and no serious symptoms have followed. The necessary precautions against sepsis were, of course, strictly observed.

1 Journ. of Mental Pathology, Oct.-Nov., 1902. 2 American Journal of Insanity, July, 1903.

\section{Progress in Dermatology.}

Howard ${ }^{1}$ has examined the pathological condition in two cases of herpes, and finds that while herpes of the trunk is associated with hæmorrhage in the posterior spinal ganglia, that affecting the upper lip and nose was associated with hæmorrhage in the Gasserian ganglion: this he claims as a new observation. Wys, Sattler, Head and Carpenter report cases of hæmorrhage in the Gasserian ganglion associated with ophthalmic herpes, and the latter two authors describe changes in the Gasserian ganglion associated with inferior maxillary herpes. The author accordingly would hold that herpes of the lip is pathologically one form of herpes zoster, and divides the forms into $(a)$ spontaneous or primary herpes, thought by Head and Carpenter, etc., to be a specific infectious disease, the specific causal agent of which has a special affinity for certain sensory ganglia (posterior spinal and Gasserian); (b) toxic herpes, dependent upon such poisons as arsenic or carbon mon-oxide; (c) that occurring with certain acute infectious diseases as pneumonia, cerebro-spinal meningitis and probably malarial and typhoid fevers. The lesions of ganglia and skin in all these are the same, and the processes therefore presumably are identical ; $(d)$ herpes simplex affecting lips and nose in coryza, gastro-intestinal intoxication, and affecting genitals. These have not been sufficiently investigated to be classified. No evidence exists for or against its connection with changes in the nervous system.

Another instance of an acute bullous eruption developing in a person whose occupation has brought him into connection with recently-killed animals, is described by Morley and Ransome. ${ }^{2}$ Cases were collected previously by Pernet and Bullock, and in most of these a small septic wound or whitlow acted as the starting point of the disease. In the case cited, a butcher aet. 26 , there was also a septic wound on the left middle finger, with a circinate scaly patch on the back of the left forearm. This latter was treated with pure formalin and dressed with white precipitate ointment, the finger being also dressed with the ointment. A slight dermatitis ensued on the treatment of the scaly patch, which extended gradually over the body, leading to a general erythema with a bullous eruption, the blebs being small. The patient ultimately recovered. The authors believe that the eruption was not due to the formalin, but was in direct association with the septic wound of the finger. Whitfield ${ }^{3}$ brings forward notes on two cases of acute pemphigus in infants and discusses the bacteriological rtiology. In the culture from one of these cases and in another previous case he has found streptococci. In the second of the reported cases no culture was made, but it was a very general case and microscopic sections showed large masses of cocci which stained well by Gram's method. Pemphigus neonatorum is now accepted as an impetiginous condition, and Whitfield asks whether these cases of acute pemphigus in infants may not be due to the same organism. In the second of his cases death took place about five hours after admission, although the child seemed to 